\section{Association between the number of prehospital defibrillation attempts and neurologic outcomes in out-of-hospital cardiac arrest patients without on- scene return of spontaneous circulation}

\author{
Seulki Choi', Tae Han Kim² ${ }^{2}$ Ki Jeong Hong ${ }^{1}$, Joo Jeong ${ }^{3}$, Young Sun Ro', \\ Kyoung Jun Song ${ }^{2}$, Sang Do Shin ${ }^{1}$
}

Department of Emergency Medicine, ${ }^{1}$ Seoul National University Hospital, Seoul, ${ }^{2}$ Seoul National University Seoul Metropolitan Government Boramae Medical Center, Seoul, ${ }^{3}$ Seoul National University Bundang Hospital, Seongnam, Korea

Objective Delivery of prehospital defibrillation for shockable rhythms by emergency medical service providers is crucial for successful resuscitation in out-of-hospital cardiac arrest (OHCA) patients. The optimal range of prehospital defibrillation attempts for refractory shockable rhythms is unknown. This study evaluated the association between the number of prehospital defibrillation attempts and neurologic outcomes in OHCA patients.

Methods A retrospective observational study was conducted using the nationwide OHCA registry. Adult OHCA patients who were treated by emergency medical service providers due to presumed cardiac origin with initial shockable rhythm were enrolled from 2013 to 2016. The final analysis was performed on patients without on-scene return of spontaneous circulation. The number of prehospital defibrillation attempts was categorized as follows: $2-3,4-5$, and $\geq 6$ attempts. The primary outcome was a good neurologic recovery at hospital discharge. Multivariate logistic regression analysis was performed to evaluate the association between neurologic outcomes and the number of prehospital defibrillation attempts.

Results A total of 4,513 patients were included in the final analysis. The numbers of patients for whom 2-3, 4-5, and $\geq 6$ defibrillation attempts were made were 2,720 (60.3\%), 1,090 (24.2\%), and 703 (15.5\%), respectively. Poorer outcomes were associated with $\geq 6$ defibrillation attempts: survival to hospital discharge (adjusted odds ratio, 0.38; 95\% confidence interval, 0.21-0.65) and good neurologic recovery (adjusted odds ratio, $0.42 ; 95 \%$ confidence interval, $0.21-0.84$ ).

Conclusion Six or more prehospital defibrillation attempts were associated with poorer neurologic outcomes in OHCA patients with an initial shockable rhythm who were unresponsive to on-scene defibrillation and resuscitation.

Keywords Defibrillators; Emergency medical services; Out-of-hospital cardiac arrest; Survival
elSSN: 2383-4625

Received: 9 June 2020

Revised: 20 July 2020

Accepted: 20 July 2020

Correspondence to: Tae Han Kim Department of Emergency Medicine, Seoul National University Seoul Metropolitan Government Boramae Medical Center, 20 Boramae-ro 5-gil, Dongjak-gu, Seoul 07061, Korea E-mail: adoong01@snu.ac.kr ORCID

https://orcid.org/0000-0003-3855-081X

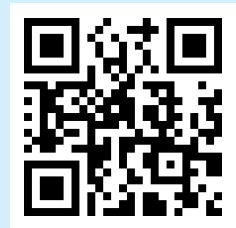

How to cite this article:

Choi S, Kim TH, Hong KJ, Jeong J, Ro YS, Song KJ, Shin SD. Association between the number of prehospital defibrillation attempts and neurologic outcomes in outof-hospital cardiac arrest patients without on-scene return of spontaneous circulation. CClin Exp Emerg Med 2021;8(1):21-29. https://doi.org/10.15441/ceem.20.069

This is an Open Access article distributed under the terms of the Creative Commons Attribution Non-Commercial License (https:// creativecommons.org/licenses/by-nc/4.0/). 


Wapsule is already known
Eummary
Early and timely defibrillation is important for out-of-hospital cardiac arrest with shockable rhythm.
What is new in the current study
An optimal range of defibrillation attempts might be associated with favorable neurologic outcome.

\section{INTRODUCTION}

High quality on-scene resuscitation by emergency medical service (EMS) providers is a key factor in improving the outcome of outof-hospital cardiac arrest (OHCA) patients. ${ }^{1,2}$ Prompt defibrillation is important for better survival and neurologic outcomes, especially in patients with an initial shockable rhythm (ventricular fibrillation [VF]/pulseless ventricular tachycardia [pVT]). Various EMS strategies have attempted to improve the provision of defibrillation in the prehospital setting. ${ }^{3-5}$ However, not all patients with an initial shockable rhythm respond to defibrillation, even after several attempts.

Previous studies of refractory VF/pVT have shown that the success rate of defibrillation is generally poor and 30-day survival rates decrease as the number of defibrillation attempts increases. $^{6,7}$ In the case of refractory VF/pVT, the secondary rescue therapy, such as administration of amiodarone and application of extracorporeal cardiopulmonary resuscitation (E-CPR), should be considered..$^{8-10}$ In many communities, especially in Asia, E-CPR and some intravenous antiarrhythmic medications are not usually provided in the prehospital setting. ${ }^{11-13}$ Therefore, if patients do not achieve a return of spontaneous circulation (ROSC) after a certain number of defibrillation attempts in the field, EMS providers must transport patients with ongoing cardiopulmonary resuscitation (CPR) in the ambulance. Determining the optimal range of defibrillation attempts before hospital transport is difficult. More defibrillation attempts on-scene may delay the secondary rescue therapy that can only be administered in the hospital. However, fewer defibrillation attempts before hospital transport risk bypassing the narrow time window in which defibrillation is highly effective because rhythm analysis and defibrillation delivery are difficult in the moving ambulance during transport to the hospital. ${ }^{14-16}$

Most EMS and CPR practice guidelines emphasize the importance of prompt rhythm analysis and on-scene delivery of defibrillation by EMS providers. However, there are no clear guide- lines regarding the optimal range of prehospital defibrillation attempts. In this study, we evaluated the association between the number of prehospital defibrillation attempts and neurologic outcomes in OHCA patients with an initial shockable rhythm. We hypothesized that an optimal range of prehospital defibrillation attempts would be associated with good neurologic recovery in OHCA patients.

\section{METHODS}

\section{Study design}

A retrospective observational study was conducted using the nationwide OHCA registry. In South Korea, a public, fire-based EMS system responds to all ambulance calls. It provides a basic to intermediate level of ambulance services, including the use of manual or automated external defibrillators and advanced airway management (e.g., endotracheal intubation and supraglottic airway insertion), under the medical direction of EMS. EMS providers are trained to check pulse and analyze rhythms using manual or automated external defibrillators after 2-minute intervals of CPR. Once defibrillation for shockable rhythms has been performed, EMS providers are trained to immediately resume chest compressions without additional pulse checks or rhythm analysis. The onscene administration of intravenous medications by EMS providers, such as epinephrine and amiodarone, is not permitted, except in extraordinary circumstances and under direct medical supervision. The EMS protocol encourages $\geq 5$ minutes of resuscitation before transport to the hospital. There are no recommendations regarding the maximum length of resuscitation time or the maximum number of on-scene defibrillation attempts when ROSC is not achieved.

The nationwide OHCA registry has been operational since 2006. It is based on a collaboration between the Korea Centers for Disease Control and Prevention and the Korean National Fire Department. The database consists of prehospital EMS records and hospital medical records. EMS run sheets were completed by on-scene 
prehospital EMS providers and stored in the Fire Department's central server. Prehospital EMS variables (ambulance call, dispatch, and arrival times; departure to hospitals; location of OHCA; medical procedures performed by emergency medical teams; and destination hospitals) and details of the EMS run sheets were reviewed by the EMS medical director responsible for each local EMS agency. Data from hospital medical records, including Utstein variables for reporting OHCA and clinical outcomes, were reviewed and extracted by certified medical reviewers from the Korea Centers for Disease Control and Prevention. Details of the data collection and handling processes of the nationwide OHCA registry have been reported previously. ${ }^{13,17,18}$

This study was approved by the relevant institutional review board (1103-153-357), which waived the requirement for informed consent.

\section{Study setting and population}

OHCA patients of presumed cardiac origin who were treated by adult EMS providers ( $\geq 18$ years of age) between January 2013 and December 2016 were initially enrolled. For the final analysis, we selected patients with an initial shockable rhythm (VF/pVT), without on-scene ROSC, who were defibrillated by EMS providers at least twice. Patients with OHCA witnessed by EMS providers and patients defibrillated by bystanders before the arrival of EMS providers were excluded. Patients with missing data regarding the number of prehospital defibrillation attempts, on-scene ROSC status, and hospital outcomes were also excluded.

\section{Study protocol}

The main exposure variable was the number of prehospital defibrillation attempts by EMS providers on-scene, categorized into three groups as follows: $2-3,4-5$, and $\geq 6$ attempts. The following prehospital EMS and hospital variables were also included in the analysis: patient age, sex, location of OHCA (private or public), bystander CPR, bystander witness status, EMS advanced airway management, EMS response time interval, EMS scene time interval, emergency department level, and provision of post resuscitation care (targeted temperature management and cardiac reperfusion therapy). Metropolitan variables included enacted metropolitan cities (Seoul, Sejong, Incheon, Ulsan, Daegu, Daejeon, Busan, and Gwangju) vs. the remaining areas of South Korea.

\section{Key outcome measures}

The primary outcome was a good neurologic recovery at hospital discharge, defined as a cerebral performance category of (1) good performance (might have mild neurologic or psychologic deficits; able to work) or (2) moderate disability (sufficient cerebral func- tion for independent activities of daily life; able to work in a sheltered environment). The other cerebral performance categories are (3) severe disability (dependent on others for daily support because of impaired brain function, ranging from ambulatory to severe dementia/paralysis), (4) coma or vegetative state, and (5) brain death. ${ }^{19}$ The secondary outcome was survival to hospital discharge.

\section{Data analysis}

Multivariate logistic regression analysis was performed to evaluate the association between neurologic outcomes and the number of prehospital defibrillation attempts. Adjustments were made for potential confounders, including age, sex, underlying medical conditions (hypertension and cardiovascular disease), location of OHCA, bystander CPR, bystander witness status, EMS response time interval, EMS advanced airway management, and EMS intravenous line insertion.

To examine differences in the effect of the number of prehospital defibrillation attempts according to the level of urbanization, interaction analysis was performed using the final multivariate logistic regression model. In South Korea, the population density is concentrated in large cities, and there are significant differences in EMS protocols between regions. We hypothesized that there would be interregional differences in hospital arrival times for OHCA patients. Therefore, we conducted an interaction analysis between metropolitan and rural areas.

Adjusted odds ratios (aORs) and 95\% confidence intervals (Cls) were estimated for survival to hospital discharge and good neurologic recovery. Statistical analyses were performed using the SAS ver. 9.4 (SAS Institute Inc., Cary, NC, USA) and Stata ver. 13.1 (Stata Corp., College Station, TX, USA).

\section{RESULTS}

A total of 10,762 adult EMS-treated OHCA patients of presumed cardiac origin with an initial shockable electrocardiographic rhythm, who were defibrillated at least twice, were initially enrolled in this study. Patients who met the following criteria: (1) OHCA witnessed by EMS providers during transport $(n=557),(2)$ defibrillated by bystanders $(n=226)$, (3) defibrillated only once $(n=3,558)$, (4) unknown number of defibrillation attempts $(n=166)$, and (5) missing outcome data $(n=2)$ were serially excluded. Of the remaining 6,253 patients, 4,513 (72.2\%) left the scene without ROSC after $\geq 2$ defibrillation attempts for shockable cardiac rhythms (Fig. 1). Patient distribution and survival outcomes are shown in Fig. 2 according to the number of prehospital defibrillation attempts by EMS providers. 


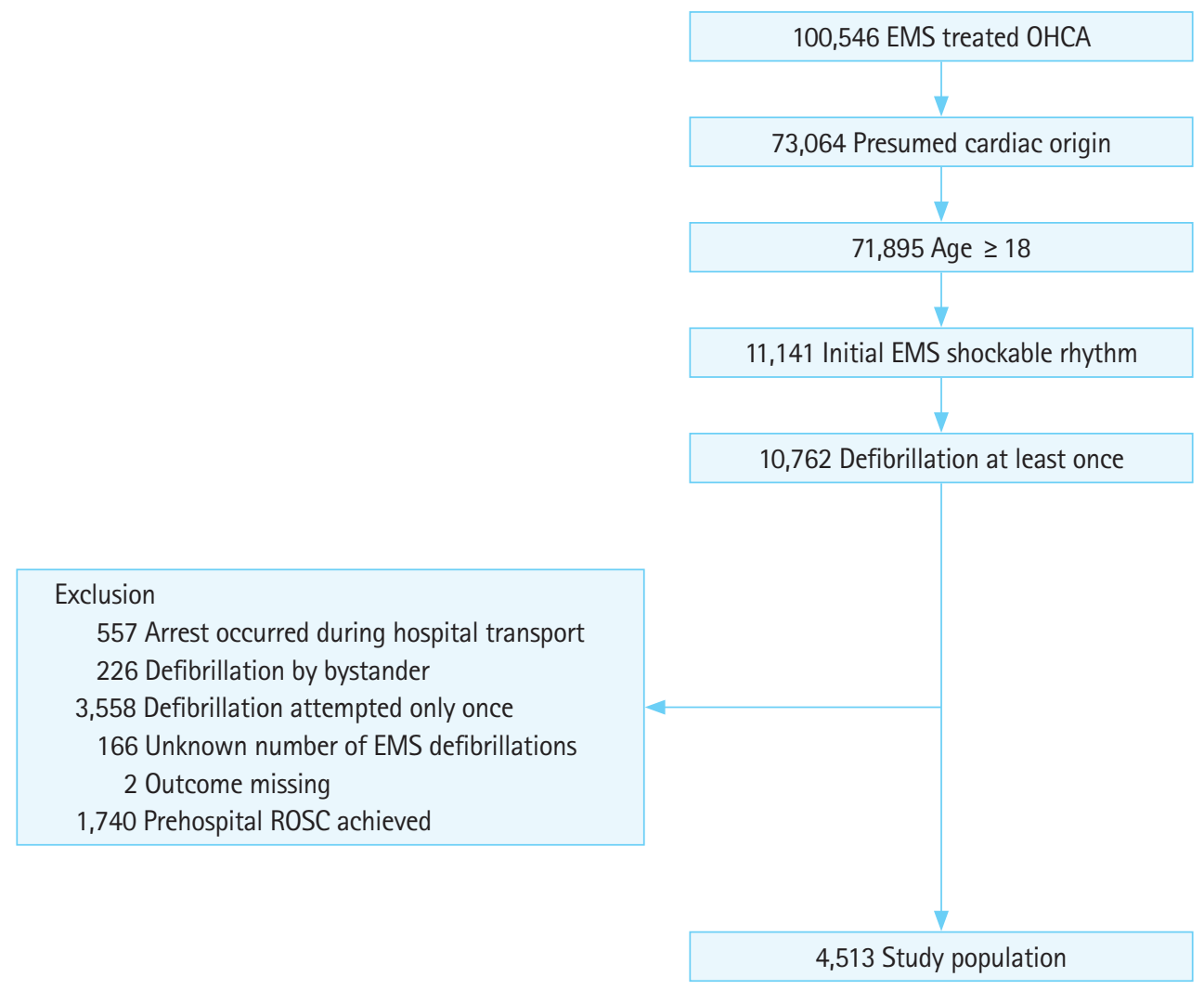

Fig. 1. Patient enrollment flowchart. EMS, emergency medical service; OHCA, out-of-hospital cardiac arrest; ROSC, return of spontaneous circulation.

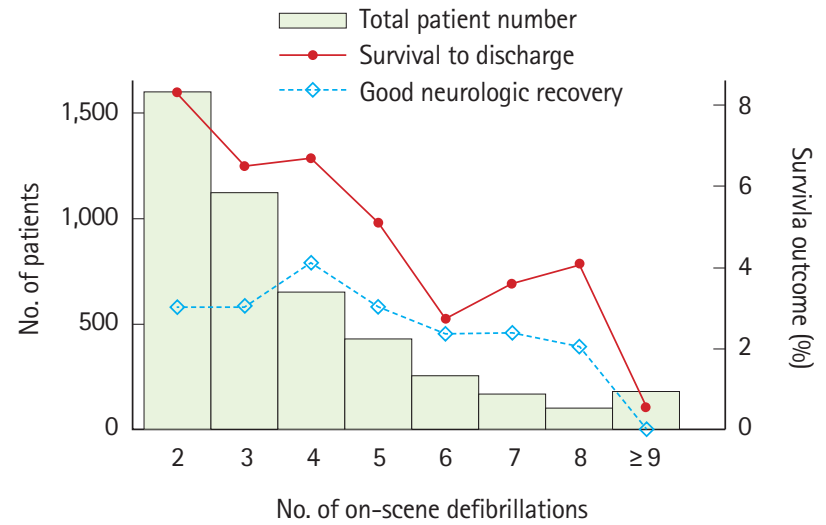

Fig. 2. Patient distribution and survival outcomes according to the number of prehospital defibrillation attempts by emergency medical service personnel in the study population.

The demographics of the study population according to the number of defibrillation attempts are shown in Table 1. Although good neurologic recovery was most frequently achieved in the group with 4-5 defibrillation attempts, the difference was not significant $(2-3,4-5$, and $\geq 6$ defibrillation attempts: $3.0 \%, 3.7 \%$, and $1.7 \%$, respectively; $P=0.06$ ).

The results of the univariate and multivariate logistic regres- sion analyses are shown in Table 2. Prehospital defibrillation attempts of $\geq 6$ were associated with poorer outcomes, both in terms of survival to hospital discharge (aOR, $0.38 ; 95 \% \mathrm{Cl}, 0.21-0.65)$ and good neurologic recovery $(\mathrm{aOR}, 0.42 ; 95 \% \mathrm{Cl}, 0.21-0.84)$.

In the interaction analysis, 2-3 defibrillation attempts $(\mathrm{aOR}$, $0.57 ; 95 \% \mathrm{Cl}, 0.35-0.93)$ and $\geq 6$ defibrillation attempts (aOR, $0.39 ; 95 \% \mathrm{Cl}, 0.17-0.90)$ were associated with significantly poorer neurologic outcomes than 4-5 defibrillation attempts in metropolitan cities (Table 3). In both metropolitan and non-metropolitan cities, survival to hospital discharge decreased as the number of prehospital defibrillation attempts increased. In non-metropolitan cities, there were no significant associations between neurologic outcomes and the number of prehospital defibrillation attempts.

\section{DISCUSSION}

We analyzed clinical outcomes according to the number of prehospital defibrillation attempts in OHCA patients with refractory shockable rhythms without on-scene ROSC. The associations between clinical outcomes and the number of prehospital defibrillation attempts were insignificant for 2-3 and 4-5 defibrillation 
Table 1. Demographics of the study population according to the number of defibrillation attempts $(n=4,513)$

\begin{tabular}{|c|c|c|c|c|}
\hline \multirow{2}{*}{ Demographic } & \multicolumn{3}{|c|}{ No. of defibrillation attempts } & \multirow{2}{*}{ P-value } \\
\hline & $2-3$ & $4-5$ & $\geq 6$ & \\
\hline Total & $2,720(60.3)$ & $1,090(24.2)$ & $703(15.5)$ & \\
\hline Year & & & & $<0.01$ \\
\hline 2013 & $741(27.2)$ & $164(15.0)$ & $69(9.8)$ & \\
\hline 2014 & $706(26.0)$ & $249(22.8)$ & $116(16.5)$ & \\
\hline 2015 & $687(25.3)$ & $323(29.6)$ & $186(26.5)$ & \\
\hline 2016 & $586(21.5)$ & $354(32.5)$ & $332(47.2)$ & \\
\hline Age (yr) & $62.7 \pm 14.7$ & $60.2 \pm 14.5$ & $58.3 \pm 14.8$ & $<0.01$ \\
\hline Male, sex & $2,148(79.0)$ & $898(82.4)$ & $595(84.6)$ & $<0.01$ \\
\hline Hypertension & $845(31.1)$ & $311(28.5)$ & $184(26.2)$ & 0.05 \\
\hline Cardiovascular disease & $621(22.8)$ & $231(21.2)$ & $145(20.6)$ & $<0.01$ \\
\hline Location of $\mathrm{OHCA}$ & & & & 0.52 \\
\hline Public & $1,073(39.4)$ & $421(38.6)$ & $261(37.1)$ & \\
\hline Private & $1,647(60.6)$ & $669(61.4)$ & $442(62.9)$ & \\
\hline Bystander CPR & $1,573(57.8)$ & $650(59.6)$ & $480(68.3)$ & $<0.01$ \\
\hline Bystander witness & $1,706(62.7)$ & $709(65.0)$ & $441(62.7)$ & 0.38 \\
\hline EMS (advanced airway management) & & & & $<0.01$ \\
\hline Endotracheal intubation & $128(4.7)$ & $96(8.8)$ & $98(13.9)$ & \\
\hline Supraglottic airway insertion & $744(27.4)$ & $456(41.8)$ & $384(54.6)$ & \\
\hline EMS (intravenous line insertion) & $392(14.4)$ & $293(26.9)$ & $299(42.5)$ & $<0.01$ \\
\hline Response time interval & $7.0(5.0-9.0)$ & $7.0(5.0-10.0)$ & $8.0(5.0-11.0)$ & $<0.01$ \\
\hline Scene time interval & $10.0(7.0-14.0)$ & $13.0(10.0-18.0)$ & $18.0(13.0-27.0)$ & $<0.01$ \\
\hline Transport time interval & $6.0(4.0-10.0)$ & $6.0(4.0-10.0)$ & $7.0(5.0-12.0)$ & $<0.01$ \\
\hline Emergency department level & & & & $<0.01$ \\
\hline Level 1 & $360(13.2)$ & $176(16.1)$ & $149(21.2)$ & \\
\hline Level 2 & $1,468(54.0)$ & $592(54.3)$ & $354(50.4)$ & \\
\hline Level 3 & $892(32.8)$ & $322(29.5)$ & $200(28.4)$ & \\
\hline Emergency department defibrillation & $1,497(55.0)$ & $630(57.8)$ & $353(50.2)$ & $<0.01$ \\
\hline Coronary reperfusion therapy & $197(7.2)$ & $59(5.4)$ & $19(2.7)$ & $<0.01$ \\
\hline Targeted temperature management & $147(5.4)$ & $38(3.5)$ & $17(2.4)$ & $<0.01$ \\
\hline \multicolumn{5}{|l|}{ Outcome } \\
\hline Survival to hospital discharge & $206(7.6)$ & $66(6.1)$ & $18(2.6)$ & $<0.01$ \\
\hline Good neurologic recovery & $82(3.0)$ & $40(3.7)$ & $12(1.7)$ & 0.06 \\
\hline
\end{tabular}

Values are presented as number (\%), mean \pm stadard deviation or median (interquartile range).

OHCA, out-of-hospital cardiac arrest; CPR, cardiopulmonary resuscitation; EMS, emergency medical service.

attempts. However, $\geq 6$ defibrillation attempts were associated with poorer survival to hospital discharge and poorer neurologic outcomes. In the interaction analysis, an intermediate number of defibrillation attempts (i.e., 4-5) was associated with better neurologic outcomes.

Our findings suggest that an excessive number of defibrillation attempts is not beneficial and may actually be harmful to patients without ROSC following serial defibrillation attempts on-scene. We believe that because of the uniqueness of our EMS system, in which intravenous medications, such as epinephrine and amiodarone, cannot be administered in the prehospital setting, delayed time to advanced cardiac life support may be one of the reasons for these findings.
In this study, patients who achieved prehospital ROSC were excluded. Patients receiving more defibrillation shocks in the field who achieved ROSC as a result of subsequent defibrillation attempts were also excluded from the final analysis. We initially expected that the outcomes of the group with 2-3 defibrillation attempts might be superior to the groups with a greater number of defibrillation attempts. However, there was no significant difference in the aOR between 2-3 and 4-5 defibrillation attempts in the total population. In the interaction analysis, 4-5 defibrillation attempts were associated with a better neurologic outcome than 2-3 defibrillation attempts in metropolitan cities. There may be several possible explanations for this. One explanation is that the number of defibrillation attempts may act as a surrogate mark- 
Table 2. Univariate and multivariate logistic regression analyses

\begin{tabular}{|c|c|c|c|c|c|}
\hline \multirow{2}{*}{ Variable } & \multirow{2}{*}{$\begin{array}{l}\text { Outcome } \\
\text { n/N (\%) }\end{array}$} & \multicolumn{2}{|c|}{ Unadjusted } & \multicolumn{2}{|c|}{ Adjusted ${ }^{\text {a) }}$} \\
\hline & & OR & $95 \% \mathrm{Cl}$ & OR & $95 \% \mathrm{Cl}$ \\
\hline \multicolumn{6}{|l|}{ Survival to hospital discharge } \\
\hline 2-3 defibrillation attempts & $206 / 2,720(7.6)$ & 1.27 & $0.95-1.69$ & 1.28 & $0.94-1.74$ \\
\hline 4-5 defibrillation attempts & $66 / 1,090(6.1)$ & 1.00 (ref) & - & 1.00 (ref) & - \\
\hline$\geq 6$ defibrillation attempts & $18 / 703(2.6)$ & 0.4 & $0.23-0.69$ & 0.38 & $0.21-0.65$ \\
\hline \multicolumn{6}{|l|}{ Good neurologic recovery } \\
\hline 2-3 defibrillation attempts & $82 / 2,720(3.0)$ & 0.81 & $0.55-1.19$ & 0.75 & $0.5-1.13$ \\
\hline 4-5 defibrillation attempts & $40 / 1,090(3.7)$ & 1.00 (ref) & - & 1.00 (ref) & - \\
\hline$\geq 6$ defibrillation attempts & $12 / 703(1.7)$ & 0.45 & $0.23-0.87$ & 0.42 & $0.21-0.84$ \\
\hline
\end{tabular}

$\mathrm{OR}$, odds ratio; $\mathrm{Cl}$, confidence interval; ref, reference; $\mathrm{EMS}$, emergency medical service.

${ }^{\text {a) }}$ Adjusted for age, sex, hypertension, cardiovascular disease, location of out-of-hospital cardiac arrest, bystander witness status, bystander-administered cardiopulmonary resuscitation, EMS response time interval, EMS advanced airway management, and EMS intravenous line insertion.

Table 3. Multivariate logistic regression and interaction analyses

\begin{tabular}{|c|c|c|}
\hline \multirow{2}{*}{ Variable } & \multicolumn{2}{|c|}{ Adjusted $^{\text {a) }}$} \\
\hline & OR & $95 \% \mathrm{Cl}$ \\
\hline \multicolumn{3}{|l|}{ Survival to hospital discharge } \\
\hline \multicolumn{3}{|l|}{ Non-metropolitan cities } \\
\hline 2-3 defibrillation attempts & 1.27 & $0.77-2.01$ \\
\hline 4-5 defibrillation attempts & 1 & - \\
\hline$\geq 6$ defibrillation attempts & 0.36 & $0.14-0.90$ \\
\hline \multicolumn{3}{|l|}{ Metropolitan cities } \\
\hline 2-3 defibrillation attempts & 1.22 & $0.83-1.78$ \\
\hline 4-5 defibrillation attempts & 1 & - \\
\hline$\geq 6$ defibrillation attempts & 0.42 & $0.21-0.83$ \\
\hline \multicolumn{3}{|l|}{ Good neurologic recovery } \\
\hline \multicolumn{3}{|l|}{ Non-metropolitan cities } \\
\hline 2-3 defibrillation attempts & 1.24 & $0.57-2.71$ \\
\hline 4-5 defibrillation attempts & 1 & - \\
\hline$\geq 6$ defibrillation attempts & 0.63 & $0.18-2.12$ \\
\hline \multicolumn{3}{|l|}{ Metropolitan cities } \\
\hline 2-3 defibrillation attempts & 0.57 & $0.35-0.93$ \\
\hline 4-5 defibrillation attempts & 1 & - \\
\hline$\geq 6$ defibrillation attempts & 0.39 & $0.17-0.90$ \\
\hline
\end{tabular}

$\mathrm{OR}$, odds ratio; $\mathrm{Cl}$, confidence interval; $\mathrm{EMS}$, emergency medical service.

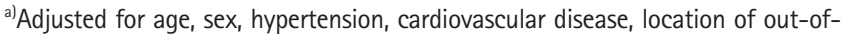
hospital cardiac arrest, bystander witness status, bystander-administered cardiopulmonary resuscitation, EMS response time interval, EMS advanced airway management, EMS intravenous line insertion.

er of sufficient on-scene resuscitation before hospital transport. During hospital transport, high-quality CPR and rhythm analysis are difficult to perform in a moving stretcher and ambulance, even for trained EMS providers. ${ }^{14-16}$ Therefore, sufficient time and effort should be given to on-scene evaluation and resuscitation before transport to the hospital. We also analyzed the association between the number of prehospital defibrillation attempts (as a continuous variable) and survival by including cubic spline. However, stratifying the number of prehospital defibrillation attempts into groups proved to be more desirable for both EMS providers and emergency room physicians.

In addition to antiarrhythmic drugs, there are other forms of management for refractory cardiac arrest that can only be provided in the hospital. One of these is the initiation of cardiopulmonary bypass during resuscitation, known as E-CPR. This involves urgent cannulation of large vessels and initiation of venoarterial extracorporeal circulation and oxygenation with an extracorporeal membrane oxygenator. Several observational studies have suggested that in conventional CPR, extracorporeal membrane oxygenation is associated with favorable outcomes in cardiac arrest patients $<75$ years of age. The International Liaison Committee on Resuscitation Advanced Life Support Task Force conducted a systemic review in $2015 .{ }^{20} \mathrm{~A}$ prospective propensity-matched observational study reported that there was a greater tendency for ROSC, as well as improved survival to hospital discharge, 30day follow-up, and 1-year follow-up (except for neurologic outcomes), in the E-CPR group than in the conventional CPR group. ${ }^{21}$ A retrospective observational study showed that $\mathrm{E}-\mathrm{CPR}$ was associated with survival and neurologic benefits at hospital discharge and 6-month follow-up. ${ }^{22}$ However, a retrospective propensitymatched observational study suggested that E-CPR was not associated with survival or neurologic benefits at hospital discharge, 30-day follow-up, or 1-year follow-up. ${ }^{23} \mathrm{~A}$ prospective observational cohort study and post hoc propensity-matched analysis showed that E-CPR was associated with better neurologic outcomes at 3-month follow-up. ${ }^{24}$ Another prospective observational study reported improved neurologic outcomes in E-CPR patients at 1- and 6-month follow-up. ${ }^{25}$ In conclusion, there is insufficient evidence to recommend the routine use of extracorporeal membrane oxygenation, which may be considered for patients with refractory cardiac arrest with shockable rhythms, in selected inclusion criteria. 
Another management strategy to support patients with refractory cardiac arrest is the administration of intravenous medications, such as epinephrine and amiodarone. Vasopressors, such as epinephrine, improve blood pressure and flow to the coronary and cerebral circulation to enhance perfusion, while antiarrhythmic drugs, such as amiodarone, facilitate the restoration and maintenance of a spontaneous perfusion rhythm in combination with the termination of VF. Several large observational studies have reported that the early use of epinephrine is associated with improved outcomes in OHCA patients. ${ }^{9,26}$ Several randomized controlled trials have also shown that patients who were administered epinephrine exhibited a higher rate of ROSC and better 30day survival than those who were administered placebo, consistent with findings from previous observational studies and a randomized controlled trial. ${ }^{27}$ Different observations have been reported regarding the use of epinephrine in patients with different electrocardiographic rhythms. Epinephrine improved the 30-day survival rate of patients with non-shockable rhythms, but no clear benefits were observed in patients with shockable rhythms. ${ }^{28,29}$ In patients with refractory shockable rhythms, antiarrhythmic drugs can help achieve successful defibrillation and reduce the risk of recurrent VF/pVT. In blinded randomized controlled trials of adult OHCA patients with refractory VF/pVT, paramedic-administered amiodarone in polysorbate (300 mg or $5 \mathrm{mg} / \mathrm{kg}$ ) improved survival to hospital admission. ${ }^{29,30}$ However, it did not affect survival to hospital discharge or neurologic outcomes. Therefore, the effectiveness of intravenous medications for patients with refractory cardiac arrest with shockable rhythms may need to be considered.

If all of the advanced cardiac and extracorporeal life support that can only be provided in the hospital could be provided onscene, then discussion regarding the optimal range of prehospital defibrillation attempts would be meaningless. However, in all EMS systems, hospital transport should be considered for patients with refractory shockable rhythms, even if the patient does not achieve ROSC. To our knowledge, there are no universal resuscitation guidelines or consensus regarding the number of prehospital defibrillations that should be attempted in patients with refractory arrhythmia before leaving the scene. We believe that the results of this study may be beneficial to EMS directors and providers who must make decisions every day between more defibrillation attempts in the field and hospital transport for OHCA patients with refractory shockable rhythms without on-scene ROSC.

There are several limitations of this study. First, the type of initial EMS electrocardiographic rhythm in the nationwide OHCA registry was recorded by emergency medical technicians. There is potential for these electrocardiographic diagnoses to be less ac- curate than those of medical doctors. In our medical oversight system, EMS medical directors reviewed all OHCA registries with scanned copies of electrocardiographic rhythm strips attached that were completed by EMS providers. Although the rate of electrocardiographic misdiagnosis has not been previously reported, the rate of confusion between shockable and non-shockable rhythms is usually very low. Second, in our nationwide OHCA registry, we did not collect the final on-scene electrocardiographic results before hospital transport. Although the enrolled patients did not achieve on-scene ROSC, it is unclear whether patients still had shockable rhythms at the commencement of hospital transport. Third, our study did not include an analysis of defibrillation times in the prehospital setting or the number of defibrillation attempts and defibrillation times after visiting the emergency room. Finally, with the exception of hospital factors, it is factors affecting ROSC in OHCA patients that are very diverse: patient age, past medical history, bystander-administered CPR and use of automated external defibrillators, EMS response time interval, EMS advanced airway management (whether successful or not), and so forth. More detailed analysis adjusting for these potential confounding variables is needed.

In summary, $\geq 6$ prehospital defibrillation attempts were associated with poorer neurologic outcomes in OHCA patients with an initial shockable rhythm who did not achieve on-scene ROSC after defibrillation by EMS providers. In metropolitan cities, 4-5 prehospital defibrillation attempts were associated with a better neurologic outcome. Future prospective studies are needed to determine the optimal range of prehospital defibrillation attempts for maximizing the neurologic outcomes of OHCA patients with refractory shockable rhythms.

\section{CONFLICT OF INTEREST}

No potential conflict of interest relevant to this article was reported.

\section{ACKNOWLEDGMENTS}

This study was supported by the Korea Centers for Disease Control and Prevention (E33010-00).

\section{REFERENCES}

1. Soar J, Nolan JP, Bottiger BW, et al. European Resuscitation Council Guidelines for Resuscitation 2015: Section 3. Adult advanced life support. Resuscitation 2015;95:100-47.

2. Kleinman $M E$, Brennan $E E_{1}$ Goldberger ZD, et al. Part 5: adult 
basic life support and cardiopulmonary resuscitation quality: 2015 American heart association guidelines update for cardiopulmonary resuscitation and emergency cardiovascular care. Circulation 2015;132(18 Suppl 2):S414-35.

3. Nelson RD, Bozeman W, Collins G, Booe B, Baker T, Alson R. Mobile versus fixed deployment of automated external defibrillators in rural EMS. Prehosp Disaster Med 2015;30:152-4.

4. Emmerson AC, Whitbread M, Fothergill RT. Double sequential defibrillation therapy for out-of-hospital cardiac arrests: the London experience. Resuscitation 2017;117:97-101.

5. Beck LR, Ostermayer DG, Ponce JN, Srinivasan S, Wang HE. Effectiveness of prehospital dual sequential defibrillation for refractory ventricular fibrillation and ventricular tachycardia cardiac arrest. Prehosp Emerg Care 2019;23:597-602.

6. Hasegawa $M$, Abe $T$, Nagata $T$, Onozuka $D$, Hagihara A. The number of prehospital defibrillation shocks and 1-month survival in patients with out-of-hospital cardiac arrest. Scand J Trauma Resusc Emerg Med 2015;23:34.

7. Holmen J, Hollenberg J, Claesson A, et al. Survival in ventricular fibrillation with emphasis on the number of defibrillations in relation to other factors at resuscitation. Resuscitation 2017; 113:33-8.

8. Kudenchuk PJ, Brown SP, Daya M, et al. Amiodarone, lidocaine, or placebo in out-of-hospital cardiac arrest. N Engl J Med 2016;374:1711-22.

9. Hayashi Y, Iwami T, Kitamura T, et al. Impact of early intravenous epinephrine administration on outcomes following outof-hospital cardiac arrest. Circ J 2012;76:1639-45.

10. Chiu CC, Chiu CW, Chen YC, Siao FY. Cardiac arrest with refractory ventricular fibrillation: a successful resuscitation using extracorporeal membrane oxygenation. Am J Emerg Med 2013;31:264. e1-2.

11. Ong ME, Shin SD, De Souza NN, et al. Outcomes for out-ofhospital cardiac arrests across 7 countries in Asia: The Pan Asian Resuscitation Outcomes Study (PAROS). Resuscitation 2015;96:100-8.

12. Ro YS, Shin SD, Kitamura T, et al. Temporal trends in out-ofhospital cardiac arrest survival outcomes between two metropolitan communities: Seoul-Osaka resuscitation study. BMJ Open 2015;5:e007626.

13. Kim TH, Shin SD, Kim YJ, Kim CH, Kim JE. The scene time interval and basic life support termination of resuscitation rule in adult out-of-hospital cardiac arrest. J Korean Med Sci 2015; 30:104-9.

14. Chan PS, Krumholz HM, Nichol G, Nallamothu BK; American Heart Association National Registry of Cardiopulmonary Resuscitation Investigators. Delayed time to defibrillation after in-hospital cardiac arrest. N Engl J Med 2008;358:9-17.

15. Kolarova J, Ayoub IM, Yi Z, Gazmuri RJ. Optimal timing for electrical defibrillation after prolonged untreated ventricular fibrillation. Crit Care Med 2003;31:2022-8.

16. White RD, Asplin BR, Bugliosi TF, Hankins DG. High discharge survival rate after out-of-hospital ventricular fibrillation with rapid defibrillation by police and paramedics. Ann Emerg Med 1996;28:480-5.

17. Ro YS, Shin SD, Lee YJ, et al. Effect of dispatcher-assisted cardiopulmonary resuscitation program and location of out-ofhospital cardiac arrest on survival and neurologic outcome. Ann Emerg Med 2017;69:52-61.

18. Park JH, Kim YJ, Ro YS, Kim S, Cha WC, Shin SD. The effect of transport time interval on neurological recovery after out-ofhospital cardiac arrest in patients without a prehospital return of spontaneous circulation. J Korean Med Sci 2019;34: e73.

19. Stiell IG, Nesbitt LP, Nichol G, et al. Comparison of the cerebral performance category score and the health utilities index for survivors of cardiac arrest. Ann Emerg Med 2009;53:241-8.

20. Callaway CW, Soar J, Aibiki M, et al. Part 4: advanced life support: 2015 international consensus on cardiopulmonary resuscitation and emergency cardiovascular care science with treatment recommendations. Circulation 2015;132(16 Suppl 1):S84-145.

21. Chen YS, Lin JW, Yu HY, et al. Cardiopulmonary resuscitation with assisted extracorporeal life-support versus conventional cardiopulmonary resuscitation in adults with in-hospital cardiac arrest: an observational study and propensity analysis. Lancet 2008;372:554-61.

22. Shin TG, Choi JH, Jo IJ, et al. Extracorporeal cardiopulmonary resuscitation in patients with inhospital cardiac arrest: a comparison with conventional cardiopulmonary resuscitation. Crit Care Med 2011;39:1-7.

23. Lin JW, Wang MJ, Yu HY, et al. Comparing the survival between extracorporeal rescue and conventional resuscitation in adult in-hospital cardiac arrests: propensity analysis of three-year data. Resuscitation 2010;81:796-803.

24. Maekawa K, Tanno K, Hase M, Mori K, Asai Y. Extracorporeal cardiopulmonary resuscitation for patients with out-of-hospital cardiac arrest of cardiac origin: a propensity-matched study and predictor analysis. Crit Care Med 2013;41:1186-96.

25. Sakamoto T, Morimura N, Nagao K, et al. Extracorporeal cardiopulmonary resuscitation versus conventional cardiopulmonary resuscitation in adults with out-of-hospital cardiac arrest: a prospective observational study. Resuscitation 2014; 85:762-8. 
26. Hagihara $A$, Hasegawa $M$, Abe $T$, Nagata $T$, Wakata $Y$, Miyazaki S. Prehospital epinephrine use and survival among patients with out-of-hospital cardiac arrest. JAMA 2012;307:1161-8.

27. Jacobs IG, Finn JC, Jelinek GA, Oxer HF, Thompson PL. Effect of adrenaline on survival in out-of-hospital cardiac arrest: a randomised double-blind placebo-controlled trial. Resuscitation 2011;82:1138-43.

28. Donnino MW, Salciccioli JD, Howell MD, et al. Time to administration of epinephrine and outcome after in-hospital cardiac arrest with non-shockable rhythms: retrospective analysis of large in-hospital data registry. BMJ 2014;348:g3028.

29. Andersen LW, Kurth T, Chase $M$, et al. Early administration of epinephrine (adrenaline) in patients with cardiac arrest with initial shockable rhythm in hospital: propensity score matched analysis. BMJ 2016;353:i1577.

30. Lin S, Callaway CW, Shah PS, et al. Adrenaline for out-of-hospital cardiac arrest resuscitation: a systematic review and meta-analysis of randomized controlled trials. Resuscitation 2014; 85:732-40. 